\title{
Preoperative Anemia and the Outcome after Coronary Artery Bypass Grafting
}

\author{
Fausto Biancari, ${ }^{1,2,3}$ Tuomas Tauriainen, ${ }^{2}$ and Eeva-Maija Kinnunen. ${ }^{2}$ \\ ${ }^{1}$ Department of Surgery, University of Turku, Turku, Finland; \\ ${ }^{2}$ Department of Surgery, University of Oulu, Oulu, Finland; \\ ${ }^{3}$ Heart Center, Turku University Hospital, Turku, Finland.
}

Running title: Anemia in coronary surgery

Keywords: Coronary artery bypass; anemia; transfusion.

Grant support: None

Conflicts of interest: None to declare

Manuscript word count: 2490 words

Abstract word count: 231 words

For correspondence:

Prof. Fausto Biancari,

Heart Center,

Turku University Hospital,

P.O. Box 5000, FI-90014 Oulu, Finland;

Tel.: +358 407333 973;

E-mail: faustobiancari@yahoo.it 
To the Editor:

Liu and Colleagues (1) kindly commented on our study evaluating the impact of preoperative anemia on the outcome after coronary artery bypass grafting (2). Their comments are of relevance since the prognostic impact of preoperative anemia and hemoglobin optimization before cardiac surgery are controversial (3).

In the present study, we adopted a propensity score matching strategy to adjust for differences between anemics and non-anemics. We do agree with Liu and Colleagues argue that propensity score analysis does not take into account a number of possible confounders. However, we included in the non-parsimonious regression model an extensive number of baseline and operative variables in order to not incur in this bias. However, we did not include early postoperative adverse events in to the regression model generating the propensity score. In fact, the aim of this analysis was to assess the effect of preoperative anemia on these adverse events. We should expect that, if anemia would have had an impact on these early adverse events, these in turn would have resulted in higher 30-day and late mortality. Furthermore, they argue that adjusting the risk of short and late mortality for the same preoperative and intraoperative confounders may be arbitrary. We do not agree on this issue as any comparative study aiming to evaluate the early and late outcome of patients should be based on cohorts with similar baseline and operative variables, therefore with the same confounders. Furthermore, both early and late mortality after adult cardiac surgery are usually associated with non-cardiac (age, gender, neurological dysfunction, extracardiac arteriopathy, renal function) and cardiac variables (left ventricular ejection fraction, recent myocardial infarction, critical preoperative state) which were taken into account in this analysis. We do agree with Liu and Colleagues that propensity score matching excluded from the analysis a significant number of non-anemics. In order to avoid this bias, we performed 
furthers analyses of the overall series. Logistic regression, linear regression, ordinal regression and Cox proportional hazards analyses were performed including the following covariates: age, gender, body mass index, platelets count, eGFR, dialysis, pulmonary disease, diabetes, stroke, extracardiac arteriopathy, neurological dysfunction, atrial fibrillation, previous percutaneous coronary intervention, previous cardiac surgery, left ventricular ejection fraction $\leq 50 \%$, recent myocardial infarction, critical preoperative status, preoperative intra-aortic balloon pump, recent ventricular arrhythmia, cardiac massage, urgency of the operation, off-pump surgery, epiaortic ultrasound, diseased ascending aorta, bilateral mammary artery grafts and radial artery graft, E-CABG bleeding classification (4) and perioperative nadir hemoglobin level. Statistical analysis were performed using SPSS statistical software (version 24.0, IBM Corporation, New York, USA). Regression models including E-CABG bleeding classification and nadir hemoglobin level confirmed that preoperative anemia was associated with prolonged intensive care unit stay and postoperative acute kidney injury (Tab. 1). Its negative impact was significant only for acute kidney injury other than new onset dialysis. Logistic regression showed that neither preoperative anemia (OR $0.71,95 \% \mathrm{CI} 0.38$-1.33) nor perioperative nadir hemoglobin (OR 0.98, 95\%CI 0.95-1.02) were associated with 30-day mortality. The E-CABG bleeding classification was an independent predictor of 30-day mortality $(\mathrm{p}<0.001$; Grade 1, OR 3.20, 95\%CI 0.88-11.72; Grade 2, OR 7.40, 95\%CI 1.90-28.75; Grade 3, OR 24.37, 95\%CI 4.70126.40).

Similarly, Cox proportional hazards analysis showed that neither preoperative anemia (HR 1.20, 95\%CI 0.97-1.48) nor perioperative nadir hemoglobin (HR 0.99, 95\%CI 0.98-1.01) were associated with late mortality. Instead, the E-CABG bleeding classification was an 
independent predictor of late mortality ( $\mathrm{p}<0.001$; Grade 1, HR 1.51, 95\%CI 1.16-1.97; Grade 2, HR 1.53, 95\%CI 1.11-2.11; Grade 3, HR 3.43, 95\%CI 2.08-5.67).

In this series of patients after coronary artery bypass grafting, blood transfusion was a powerful predictor of early and late mortality, whereas the negative impact of preoperative anemia was limited to postoperative acute kidney injury.

\section{References}

1. Liu YY, Xue FS, Li HX (2017) Assessing effects of preoperative anemia on adverse outcomes after coronary surgery. World J Surg (in press)

2. Tauriainen T, Koski-Vähälä J, Kinnunen EM, et al (2017) The Effect of preoperative anemia on the outcome after coronary surgery. World J Surg 41:1910-1918. 3. D'Ambra MN, FitzGerald D, Kaufman RM, Shekar P (2015) Blood conservation: Why aren't we doing this for everyone? J Thorac Cardiovasc Surg 150:984-985.

4. Biancari F, Ruggieri VG, Perrotti A, et al (2015) European multicenter study on coronary artery bypass Grafting (E-CABG registry): study protocol for a prospective clinical registry and proposal of classification of postoperative complications. J Cardiothorac Surg 10:90. 
Table 1. Outcome in the overall series according to univariate and multivariate analysis.

\begin{tabular}{|c|c|c|c|c|c|}
\hline & $\begin{array}{c}\text { Overall } \\
\text { population } \\
\text { No. } 2761\end{array}$ & $\begin{array}{c}\text { No preop. } \\
\text { anemia } \\
\text { No. } 2099\end{array}$ & $\begin{array}{c}\text { Preop. } \\
\text { anemia } \\
\text { No. } 662 \\
\end{array}$ & $\begin{array}{c}\text { Univariate } \\
\text { analysis } \\
P \text {-value }\end{array}$ & $\begin{array}{c}\text { Multiple covariates } \\
\text { adjusted analysis }\end{array}$ \\
\hline 30-day mortality & $89(3.2)$ & $48(2.3)$ & $41(6.2)$ & $<0.0001$ & $0.71,0.38-1.33$ \\
\hline ICU stay (days) & $2.2 \pm 2.5$ & $1.9 \pm 2.0$ & $2.9 \pm 3.5$ & $<0.0001$ & $1.53,1.17-2.01$ \\
\hline Stroke & $58(2.1)$ & $33(1.6)$ & $25(3.8)$ & 0.001 & $1.12,0.59-2.17$ \\
\hline Atrial fibrillation & $1185(42.9)$ & $848(40.4)$ & $337(50.9)$ & $<0.0001$ & $0.96,0.77-1.20$ \\
\hline Ventricular fibrillation/asystole & $48(1.7)$ & $24(1.1)$ & $24(3.6)$ & $<0.0001$ & $1.37,0.66-2.84$ \\
\hline Postop. intra-aortic balloon pump & $16(0.6)$ & $11(0.5)$ & $5(0.8)$ & 0.556 & $0.61,1.15-2.54$ \\
\hline Repeat CABG or PCI & $14(0.5)$ & $10(0.5)$ & $4(0.6)$ & 0.753 & $0.88,0.20-3.90$ \\
\hline Post-operative use of antibiotics & $935(33.9)$ & $613(29.2)$ & $322(48.6)$ & $<0.0001$ & $1.14,0.91-1.42$ \\
\hline Deep SWI/mediastinitis & $68(2.5)$ & $46(2.2)$ & $22(3.3))$ & 0.101 & $0.75,0.40-1.42$ \\
\hline Surgery for gastrointest. compl. & $32(1.2)$ & $16(0.8)$ & $16(2.4)$ & 0.001 & $0.79,0.28-2.26$ \\
\hline Acute kidney injury & & & & $<0.0001$ & $-0.32,-0.58--0.05$ \\
\hline Grade 1 & $325(12.0)$ & $181(8.7)$ & $144(23.0)$ & & \\
\hline Grade 2 & $71(2.6)$ & $40(1.9)$ & $31(4.9)$ & & \\
\hline Grade 3 & $61(2.3)$ & $33(1.6)$ & $28(4.5)$ & & \\
\hline $\begin{array}{l}\text { Acute kidney injury without } \\
\text { dialysis }\end{array}$ & $396(14.3)$ & $223(10.6)$ & $173(26.1)$ & $<0.0001$ & $1.53,1.17-2.01$ \\
\hline New renal replacement therapy & $51(1.9)$ & $28(1.3)$ & $23(3.6)$ & $<0.0001$ & $0.57,0.25-1.31$ \\
\hline Survival** & & & & $<0.0001$ & $1.20,0.97-148$ \\
\hline 1 -year & & $96.0 \%(2015)$ & $88.7 \%$ (587) & & \\
\hline 5-year & & $88.4 \%$ (1287) & $72.7 \%(319)$ & & \\
\hline 8 -year & & $80.2 \%(471)$ & $62.4 \%(82)$ & & \\
\hline
\end{tabular}

Risk estimates in bold indicate statistical significance in multivariate analysis. Continuous variables are reported as mean and standard deviation. Categorical variables are reported as counts and percentages. ICU: intensive care unit; $\mathrm{CABG}$ : coronary artery bypass grafting; PCI: percutaneous coronary intervention; SWI: sternal wound infection; Risk estimates are odds ratio or beta coefficient; 95\%CI: $95 \%$ confidence interval; *: excluding bleeding-related outcomes; **: in parentheses are patients at risk. 